\title{
Endocarditis due to Multidrug-Resistant Klebsiella pneumoniae-KPC
}

\author{
Fermin Lopez-Rivera ${ }^{\mathrm{a}, \mathrm{c}}$, Hernan Gonzalez Monroiga ${ }^{\mathrm{a}}$, Omar Mendez Melendez ${ }^{\mathrm{a}}$, \\ James Eggert ${ }^{\mathrm{b}}$, Fernando Abreu ${ }^{\mathrm{a}}$
}

\begin{abstract}
Infective endocarditis (IE) is defined as a microbial infection of the cardiac endothelium, commonly involving, but not limited to, the cardiac valves. This pathology could lead to dire complications, carrying a high morbidity and a 6 -month mortality of $25 \%$. Its incidence increases proportionally with age and presents with a bimodal distribution and a frequency of 1/100,000 in young adults and 10/100,000 in patients older than 75 years. IE can be classified as native valve endocarditis or prosthetic valve endocarditis, with the former having higher incidence rates. Additionally, IE can be named as either rightsided or left- sided endocarditis, with each one displaying unique clinical features. We report a case of a 42-year-old Hispanic male patient with a medical history of intravenous drug abuser (IVDA), hepatitis $\mathrm{C}(\mathrm{HCV})$ and chronic alcohol abuse that arrived at the emergency room with an unquantified fever, myalgia and nausea for 4 days before admission. Patient was admitted to the medicine ward with a diagnosis of fever of unknown origin, hypernatremia and dehydration. Patient was initially treated with vancomycin and cefepime and hydration but after $72 \mathrm{~h}$ patient persisted with fever (average $38.8^{\circ} \mathrm{C}$ ), developed a rash on the palms of the hands and the soles of the feet and a new 2/6 murmur was noticed at the mitral area. Blood culture recovered multidrug-resistant Klebsiella pneumoniae with Klebsiella pneumoniae carbapenemase (KPC), and therapy was switched to polymyxin $\mathrm{B}$ and high-dose extended-infusion meropenem for 42 days. The patient improved and underwent valve replacement 8 weeks after discharge. IE must remain high on the differential diagnosis in every patient with fever of unknown origin. The physical exam must also remain as the cornerstone for diagnosis, as in this patient, where the development of a new hand rash and a new murmur altered the diagnosis, prognosis and management. IE poses a high mortality rate from common pathogens and is a difficult pathology to handle. The management of endocarditis due to a KPC is a real challenge in view of limited data and limited antibiotic options.
\end{abstract}

Keywords: Endocarditis; Klebsiella pneumoniae-KPC; Dukes criteria

Manuscript submitted September 9, 2017, accepted September 18, 2017

anternal Medicine Department, San Juan City Hospital, San Juan, Puerto Rico bSan Juan Bautista Medicine School, Caguas, Puerto Rico

${ }^{\mathrm{c} C o r r e s p o n d i n g ~ A u t h o r: ~ F e r m i n ~ L o p e z-R i v e r a, ~ I n t e r n a l ~ M e d i c i n e ~ D e p a r t m e n t, ~}$ San Juan City Hospital, 550 Calle Jazmin Coto Laurel, San Juan 00780, Puerto Rico. Email: drlopezrivera.ga@gmail.com

doi: https://doi.org/10.14740/jmc2912w

\section{Introduction}

Infective endocarditis (IE) is defined as an infection of the heart valves or cardiac endothelium with symptoms that vary considerably, ranging from subtle and non-specific to catastrophic [1]. It is an uncommon entity that leads to serious morbidity and mortality, if not diagnosed promptly [2]. The incidence has increased during the last decades and rises proportionally to age, presenting with a bimodal distribution and a frequency of 1/100,000 in young adults and 10/100,000 in patients older than 75 years [3]. Despite the low incidence and significant advances, IE poses a high 6-month mortality rate of $25 \%$ [4]. IE can be classified as native valve endocarditis (NVE) or prosthetic valve endocarditis (PVE), where NVE is affected more frequently than PVE with a ratio of 2.3:1 [5]. In addition, it can be classified as right-sided and left-sided endocarditis, where right-sided endocarditis accounts for $10 \%$ of all cases with a high prevalence among IV drug users [6]. The most prevalent clinical findings of right-sided endocarditis are: chest pain, shortness of breath, cough, hemoptysis, and pulmonary emboli. Left-sided endocarditis involves more peripheral embolic events including: upper and lower extremities, mycotic aneurism and strokes [6]. The most commonly affected valves are the mitral valve, aortic valve, tricuspid and pulmonary valve. To diagnose IE, a patient must meet the Duke criteria, where it is divided into two groups: major criteria and minor criteria (Table 1). Endocarditis is classified in one of three diagnostic categories based on the Duke criteria: definitive IE (two major criteria, one major and three minor criteria or five minor criteria), possible infective (three minor criteria) and rejected IE [7]. The most common isolated pathogen in IE is Staphylococcus aureus, whereas non-HACEK group is responsible for less than $1.8 \%$ [8]. Klebsiella pneumoniae is a rare agent in regard to IE and is classified as non-HACEK group [9]. Klebsiella pneumoniae carbapenemase (KPC) is an emerging problem because of highly drug-resistant bacteria that limit antibiotic choice and can be identified with genetic testing but, when unavailable, sensitivity resistance to ertapenem is a good indicator of KPCs [10].

\section{Case Report}

A case of a 42-year-old Hispanic male patient with a medical history of intravenous drug abuse (IVDA), hepatitis C (HCV) 
Table 1. Duke Criteria

\begin{tabular}{ll}
\hline Major criteria & Positive blood culture \\
& Classic echocardiogram findings \\
Minor criteria & Predisposing factors (poor dentition, IVDA) \\
& Fever (over $\left.38.0^{\circ} \mathrm{C}\right)$ \\
& Vascular phenomena (hemorrhages, embolic) \\
& Immunologic phenomena (glomerulonephritis, Osler's nodes) \\
\hline
\end{tabular}

and chronic alcohol abuse that arrived at the emergency room with an unquantified fever, myalgia and nausea for 4 days before admission. Patient was admitted to the medicine ward with a diagnosis of fever of unknown origin, hypernatremia and dehydration. Triage vital signs showed blood pressure of 115/75 mm Hg, heart rate of 102 beats per minute, respiratory rate of 21 per minute, temperature of $38.6{ }^{\circ} \mathrm{C}$, and pulse oximetry of $96 \%$ on room air. Physical exam was remarkable for an acutely ill patient with poor dentition and no additional findings (including lungs clear to auscultation and no heart murmurs noted). Initial laboratory studies were remarkable for white blood cells of $23.73 \times 10^{9} / \mu \mathrm{L}$, hemoglobin of 8.9 $\mathrm{g} / \mathrm{dL}$, hematocrit of $26.8 \%$, platelets of $408 \times 10^{9} / \mathrm{L}$, sodium of $151 \mathrm{mmol} / \mathrm{L}$, potassium of $3.6 \mathrm{mmol} / \mathrm{L}$, chloride of 119.00 $\mathrm{mmol} / \mathrm{L}$, carbon dioxide of $28.00 \mathrm{mmol} / \mathrm{L}$, blood urea nitrogen of $16 \mathrm{mg} / \mathrm{dL}$, creatinine of $0.74 \mathrm{mg} / \mathrm{dL}$, glomerular filtration rate by Cockcroft-Gault of $108.5 \mathrm{~mL} / \mathrm{min}$, blood urea nitrogen/ creatinine ratio of 21.62 , C-reactive protein of $32 \mathrm{mg} / \mathrm{dL}$, and erythrocyte sedimentation rate of $72 \mathrm{~mm} / \mathrm{h}$ and urine analysis was remarkable for red blood cells 25 - 30 per HPF. Chest Xray was completely unremarkable. Patient was initially treated with vancomycin $1 \mathrm{~g}$ IV every $12 \mathrm{~h}$ and cefepime $2 \mathrm{~g}$ IV every $8 \mathrm{~h}$ (three blood cultures were ordered $1 \mathrm{~h}$ apart and the first was obtained before the start of the antibiotic) and hydration. After $72 \mathrm{~h}$, the patient persisted with fever (average $38.8^{\circ} \mathrm{C}$ ), developed a painless rash on the palms of the hands and the soles of the feet soles (Janeway lesions), along with splinter hemorrhages and a new 2/6 murmur was noticed at the mitral valve area that increased with expiration (Table 2; Figs. 1-3). Echocardiogram was performed and showed mitral valve vegetation with regurgitation (Fig. 4). Blood culture recovered multidrug-resistant Klebsiella pneumoniae-KPC (three/ three bottles) with minimal inhibitory concentration (MIC) $>$ 2 for ertapenem, and therapy was switched to polymyxin B 750,000 units IV every $12 \mathrm{~h}$ and meropenem $2 \mathrm{~g}$ IV to over $4 \mathrm{~h}$ every $8 \mathrm{~h}$ (high-dose extended-infusion) for 42 days. The patient became afebrile in the next $48 \mathrm{~h}$ and blood cultures were repeated (three blood cultures $1 \mathrm{~h}$ apart) and final report showed no growth. During the hospitalization, cardiothoracic surgery staff was consulted on the patient case and recommended valve replacement 8 weeks after completing antibiotic therapy. Finally, patient underwent mitral valve replacement with no complications and has been monitored at the internal medicine clinic.

\section{Discussion}

The patient arrived at the emergency room complaining of vague symptoms such as fever with no apparent foci. In view of prominent leukocytosis and high-risk behaviors, the patient was started on broad-spectrum antibiotics with antipseudomonal coverage. Although upon initial evaluation (triage and labs), the patient met three minor Duke criteria: fever, predisposing factors and immunologic phenomena (nephrotic range microscopic hematuria), and endocarditis was not considered. The three predominant risk factors for IE are: IVDA, implantable cardiac device (such as a pacemaker) and an unrepaired congenital heart disease [11]. However, the prescribed antibiotics in this case provide coverage against most common pathogens [8]. The physical findings of rash and murmur $(2 / 6$ on Levine scale) at $72 \mathrm{~h}$ changed course of the hospitalization. The echocardiogram and other tests, such as rheumatoid factor, strengthened the suspicion of IE $[12,13]$. The echocardiogram revealed a large vegetation; however, a negative transthoracic echocardiogram does not rule out IE, since only $25 \%$ of vegetations less that $5 \mathrm{~mm}$ are identified [14]. Three sets of blood cultures were obtained and all of them recovered Klebsiella pneumoniae. The patient met one major criterion and five minor criteria of the Duke criteria: classic echocardiographic findings (major), predisposing factors, immunologic phenomena, fever, non-classic blood culture, and vascular phenomena. Blood culture sensitivity showed an elevated MIC for ertapenem, a marker for KPCs, as a meropenem disk was not available

Table 2. Levine Scale for Murmurs

\begin{tabular}{ll}
\hline Grade 1 & Faint, heard only with special effort \\
Grade 2 & Faint, but is immediately audible \\
Grade 3 & Moderately loud, no thrill \\
Grade 4 & Loud murmur, no thrill \\
Grade 5 & Extremely loud, audible with one edge of the stethoscope touching the chest wall \\
Grade 6 & Extremely loud, heard without a stethoscope \\
\hline
\end{tabular}




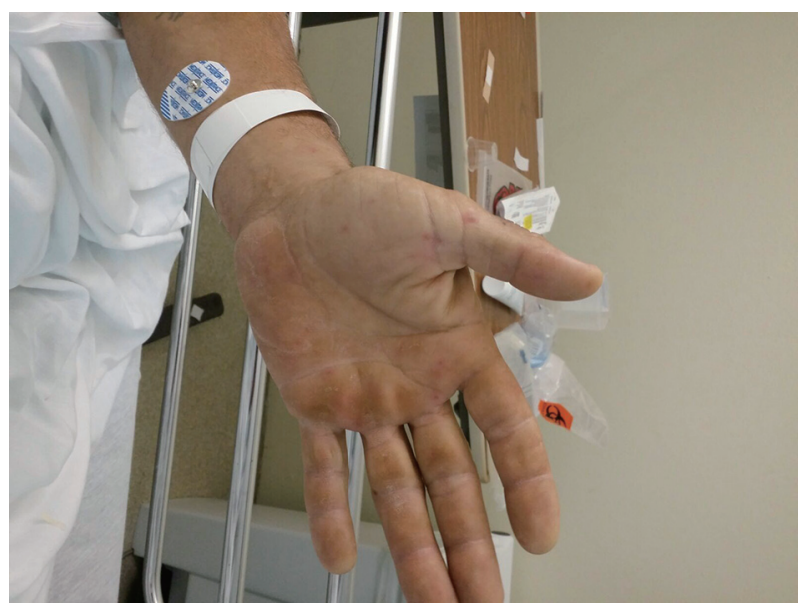

Figure 1. Hand Janeway lesions.

for sensitivity; however, this novel drug was used in high-dose extended infusion combined with polymyxin B as a salvage therapy, resulting in a positive outcome.

\section{Conclusion}

This case presented several challenging and unexpected aspects. First, IE due to IVDA often affects the tricuspid valve. Negative findings of the classic right-sided endocarditis do not

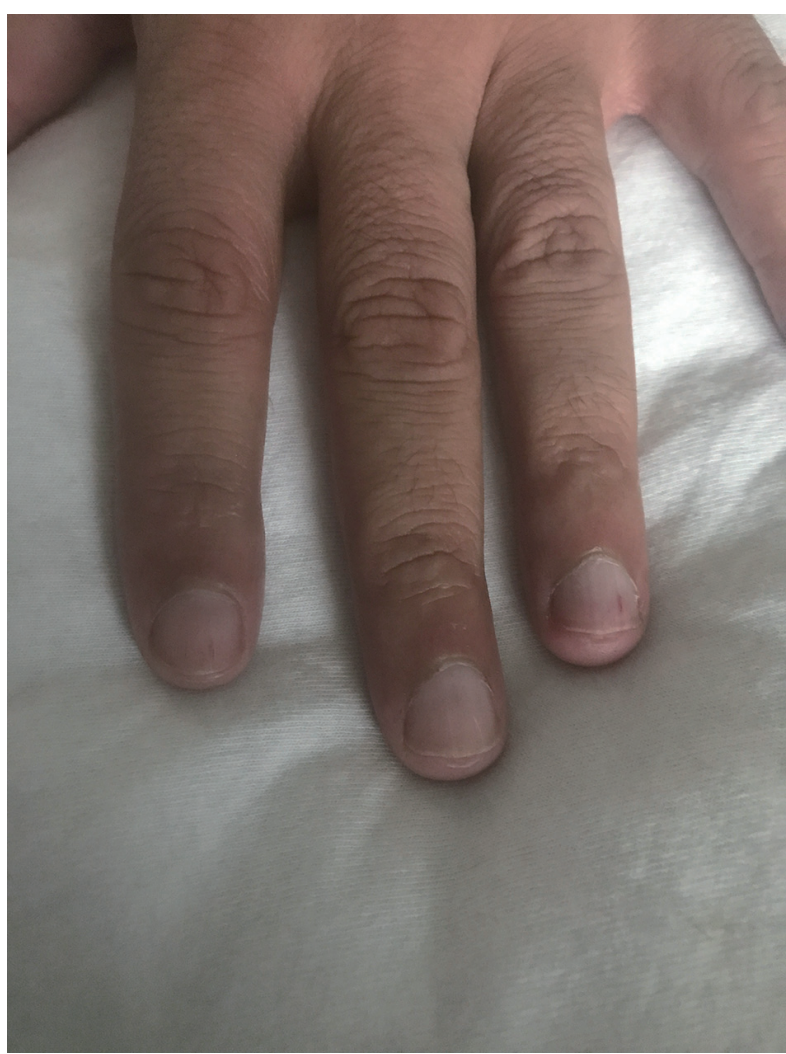

Figure 2. Splinter hemorrhages.

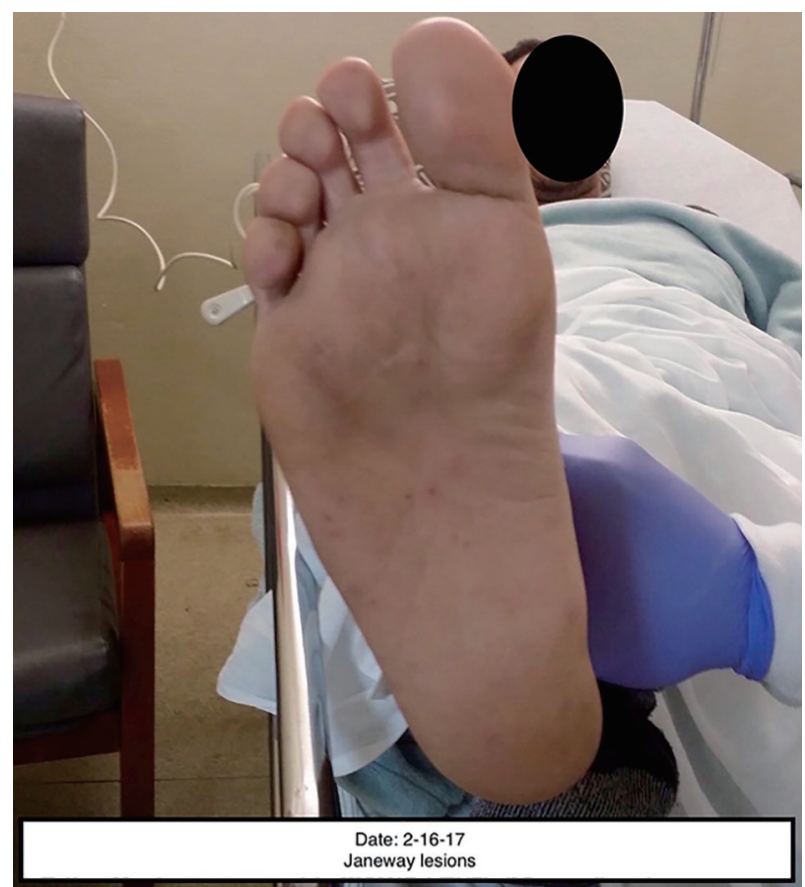

Figure 3. Foot Janeway lesions.

rule out this pathology, as occurred in our case, an IVDA patient with mitral valve involvement. Secondly, we would normally expect IE due to Pseudomonas aeruginosa in an IVDA patient, but our pathogen was determined to be Klebsiella pneumoniae, a pathogen responsible for less than $1.8 \%$ of IE cases. Third, blood cultures revealed multidrug-resistant bacteria, resistant to ceftazidime and ertapenem, characteristic of a KPC [15]. This report is limited in that we could not perform a confirmatory KPC test due to the lack of availability of this test. The isolate was resistant to ceftazidime and ertapenem, and based

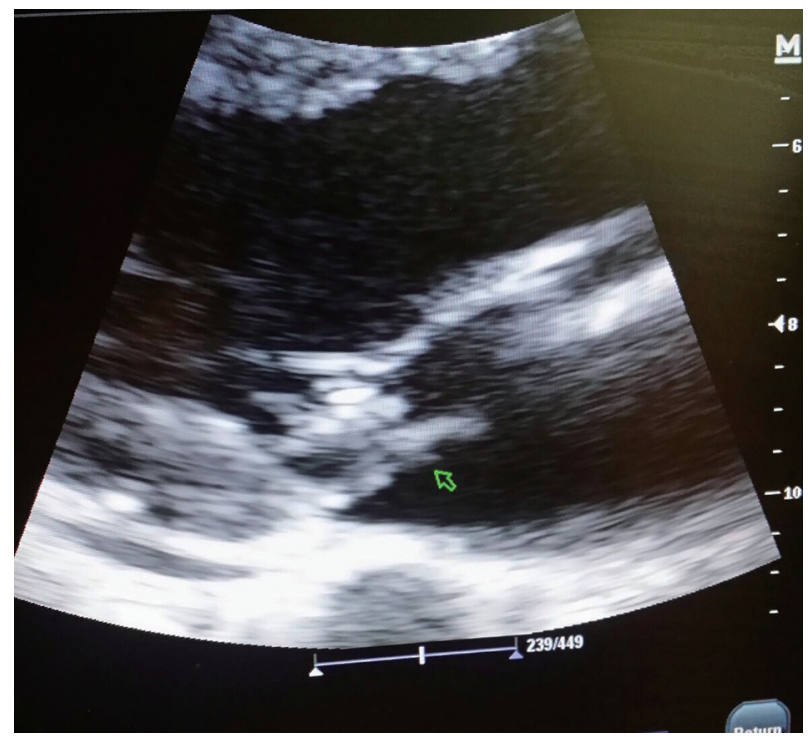

Figure 4. Flail posterior leaflet with moderate regurgitation and vegetation $1.6 \times 0.6 \mathrm{~cm}$. 
on that finding, the pathogen was managed as a KPC. This case presents successful treatment in left-sided IE due to multidrugresistant Klebsiella pneumoniae-KPC secondary to IVDA with the combination of meropenem and polymyxin B.

\section{Acknowledgments}

The authors wish to acknowledge the guidance and assistance of Emily Pagan Colon, RN, in the preparation of the manuscript of this article.

\section{Financial Support}

No source of financial support to disclose.

\section{Conflict of Interest}

We have no conflict of interest to declare.

\section{References}

1. Wu Wei Tsung, Hung-Ling Huang, Ho-Ming Su, TsungHsien Lin, Kun-Tai Lee. Supraventricular tachycardia as the initial presentation of bacterial infective endocarditis: A rare case report. Case Reports in Internal Medicine 2015;2.3

2. Paterick TE, Paterick TJ, Nishimura RA, Steckelberg JM. Complexity and subtlety of infective endocarditis. Mayo Clin Proc. 2007;82(5):615-621.

3. Owens Thomas A, Vance G. Fowler, Edward F. Pilkington. Infective Endocarditis. Principles and practice of hospital medicine. 2nd ed. New York: McGraw Hill, 2016, p. 1-639.

4. Hasbun R, Vikram HR, Barakat LA, Buenconsejo J, Quagliarello VJ. Complicated left-sided native valve endo- carditis in adults: risk classification for mortality. JAMA. 2003;289(15):1933-1940.

5. Male KR, Mathews A, Mower J. An unusual presentation of an unusual disease: infective endocarditis: a case report and review of the literature. Cases J. 2008;1(1):292.

6. Moss R, Munt B. Injection drug use and right sided endocarditis. Heart. 2003;89(5):577-581.

7. Topan A, Carstina D, Slavcovici A, Rancea R, Capalneanu R, Lupse M. Assesment of the Duke criteria for the diagnosis of infective endocarditis after twenty-years. An analysis of 241 cases. Clujul Med. 2015;88(3):321-326.

8. McDonald JR. Acute infective endocarditis. Infect Dis Clin North Am. 2009;23(3):643-664.

9. Anderson MJ, Janoff EN. Klebsiella endocarditis: report of two cases and review. Clin Infect Dis. 1998;26(2):468474.

10. Thipperudraswamy T, Sudhindra K.s, Nadagir D. Shobha, Mahesh Kumar. Emergence of Klebsiella pneumoniae Carbapenemase (KPC)-Producing Klebsiella pneumoniae from clinical isolates in tertiary care center in $\mathrm{BMCH}$, Chitradurga. International Journal of Medical Science and Clinical Invention. 2015.

11. Yuan SM. Right-sided infective endocarditis: recent epidemiologic changes. Int J Clin Exp Med. 2014;7(1):199218.

12. Alpert MA. Systolic Murmurs. In: Walker HK, Hall WD, Hurst JW, editors. Clinical methods: the history, physical, and laboratory examinations. 3rd edition. Boston: Butterworths; 1990. Chapter 26.

13. Silverman ME, Wooley CF. Samuel A. Levine and the history of grading systolic murmurs. Am J Cardiol. 2008;102(8):1107-1110.

14. Gonzalez-Alujas, Maria Teresa, Artur Evangelista Masip. Echocardiography in Infective Endocarditis Diagnosis. Infective Endocarditis. 2016:37-50.

15. Arnold RS, Thom KA, Sharma S, Phillips M, Kristie Johnson J, Morgan DJ. Emergence of Klebsiella pneumoniae carbapenemase-producing bacteria. South Med J. 2011;104(1):40-45. 